\title{
O Cuidado de Enfermagem no Atendimento de Emergências Oncológicas: uma revisão
}

\author{
integrativa
}

\author{
The Nursing Care at Emergency Cancer Care: an integrative review
}

\section{Luciano Godinho Almuinha Ramos ${ }^{1}{ }^{*}$ Vera Maria Sabóia ${ }^{2}$ Rafael Gravina Fortini ${ }^{3}$}

\begin{abstract}
Resumo
Este estudo versa sobre a assistência de enfermagem nas emergências oncológicas. Objetiva-se destacar o conhecimento sobre o cuidado de enfermagem no atendimento a pessoas que vivem com neoplasias e encontram-se em situação de emergência oncológica. Trata-se de uma revisão integrativa com a seguinte questão norteadora: como se dá o cuidado de enfermagem numa emergência oncológica, tendo em vista o aprimoramento da assistência? A busca das publicações foi realizada nas bases de dados LILACS, PUBMED e BVS. Como critério de inclusão, foram selecionados artigos publicados entre os anos de 2007 e 2016, utilizando-se os seguintes descritores: Enfermagem em Emergência, Cuidados de Enfermagem e Neoplasias. O conhecimento evidenciado nas publicações selecionadas mostrou-se reduzido. Todavia, entre os artigos de enfermagem relativos às emergências oncológicas, a maioria limitou-se a descrever as intervenções específicas para cada situação, tais como: observação do paciente, favorecimento do conforto físico, orientações ao paciente e seus familiares. Conclui-se a necessidade de um maior incentivo para estudos nesta temática, para que os enfermeiros estejam cada vez mais capacitados e embasados em princípios científicos, contribuindo para um melhor desempenho da prática profissional.
\end{abstract}

Palavras-chave: Enfermagem em Emergência; Cuidados de Enfermagem; Neoplasias.

\section{Abstract}

This study deals with nursing care in oncologic emergencies. The aim is to highlight the knowledge about nursing care to people living with neoplasms and who are in an oncologic emergency situation. This is an integrative review with the following guiding question: how does nursing care take place in an oncologic emergency, bearing in mind the enhancement of care? The search of the publications was performed in the LILACS, PUBMED and BVS databases. Concerning the inclusion criteria, we selected papers published between 2007 and 2016, using the following descriptors: Emergency Nursing, Nursing Care and Neoplasms. The knowledge evidenced in the selected publications proved to be reduced. Nevertheless, among the nursing papers related to oncologic emergencies, most were limited to describing specific nursing interventions for each situation, such as observing the patient, favoring of the physical comfort, guidance to the patient and to their family members. In conclusion, there is a need for greater encouragement for this field of research, so that the nurses are increasingly trained and based on scientific principles, thus achieving a good performance in their function.

Keywords: Emergency Nursing; Nursing Care; Neoplasms.

1- Enfermeiro. Mestrando em Enfermagem da Escola de Enfermagem da Universidade Federal Fluminense - UFF. Niterói, RJ - Brasil. Oficial Enfermeiro. Coordenador do Curso de Aperfeiçoamento em Enfermagem da Escola de Enfermagem do Hospital Naval Marcílio Dias - Marinha do Brasil (lucianogodinho@yahoo.com.br). Endereço para correspondência: Avenida Governador Celso Peçanha, 250, Apt. 101. Bairro: Centro. Município: Mesquita- RJ. CEP: 26551200.

2 - Enfermeira. Professora Titular da Disciplina de Fundamentos de Enfermagem da Escola de Enfermagem da Universidade Federal Fluminense - UFF. Niterói, RJ - Brasil (verasaboia@uol.com.br).

3 - Enfermeiro. Mestrando em Enfermagem da Escola de Enfermagem da Universidade Federal Fluminense - UFF. Niterói, RJ - Brasil (rafagravina@gmail.com). 


\section{Introdução}

As causas do câncer são variadas, sendo elas internas e externas, podendo ou não, estar interligadas. As causas externas referem-se ao meio ambiente, aos hábitos e costumes de uma sociedade. Já causas internas, relacionam-se à capacidade de defesa do organismo a agressões externas, fatores genéticos, psicológicos, reprodutivos, ambientais, hábitos de vida, irradiação e vírus.

O câncer é responsável por mais de $12 \%$ de todas as causas de óbitos no mundo, mais de 7 milhões de pessoas morrem a cada ano após um diagnóstico oncológico. 0 Sistema Único de Saúde aumentou sua capacidade para atender essa clientela, a fim de conseguir um diagnóstico o mais precoce possível(1).

No Brasil, o aumento da incidência de câncer ocorre pela maior exposição dos indivíduos aos agentes cancerígenos. Mudança no estilo de vida, nutrição e consumo desencadeados pelo processo global de industrialização, têm reflexos importantes na epidemiologia das populações. $\mathrm{O}$ aumento da expectativa de vida e o envelhecimento populacional aumentaram a incidência de doenças crônico-degenerativas, assim como o câncer(2).

Assim, esta pesquisa bibliográfica tem como objetivo: destacar o conhecimento sobre o cuidado de enfermagem no atendimento a pessoas que encontram-se em situação de emergência oncológica.

\section{Método}

Trata-se de uma pesquisa, do tipo revisão integrativa, com a seguinte questão norteadora: como se dá o cuidado de enfermagem numa emergência oncológica, tendo em vista o aprimoramento da assistência?

Para a seleção de artigos, foram acessadas as seguintes bases de dados: Literatura Latino-Americana e do Caribe em Ciências da Saúde (LILACS), Biblioteca Virtual em Saúde (BVS) e BVS Oncologia. Foram selecionados artigos publicados entre os anos de 2007 e 2016. Este recorte temporal deu-se devido ao período de construção e realização de uma especialização em oncologia clínica, no município do Rio de Janeiro. Foram incluídos artigos completos, disponíveis online, publicados em português, inglês e espanhol. Foram excluídos artigos que não abordavam a temática em questão e com textos repetidos.

A fim de obter o maior número de artigos sobre a temática escolhida, foram elaboradas estratégias de busca nas bases de dados, utilizando o cruzamento dos descritores Enfermagem em Emergência 
AND Neoplasias. Foram encontradas 246 produções, sendo 153 disponíveis. Em seguida, cruzamos também os descritores Enfermagem em Emergência AND Cuidados de Enfermagem AND Neoplasias, foram selecionados 9 artigos que atendiam os critérios adotados, para leitura na íntegra e posterior discussão.

Outra busca foi realizada na base de dados das Publicações Médicas (PUBMED), na qual encontram-se publicados apenas artigos em inglês. Nesta pesquisa foram encontrados 3.093 publicações. Quando utilizou-se o cruzamento dos Mesh Terms Emergency Nursing AND Nursing Care AND Neoplasms, foram encontrados 210 artigos. Excluiramse artigos repetidos e que não abordavam a temática do estudo. Ao final, encontraramse 9 artigos que foram adicionados ao quadro para discussão.

A seguir, um fluxograma que sintetiza a busca dos 18 artigos que compuseram a amostra final da revisão.

Fluxograma 1. Busca e seleção dos 18 artigos. Rio de Janeiro, RJ, Brasil, 2017.

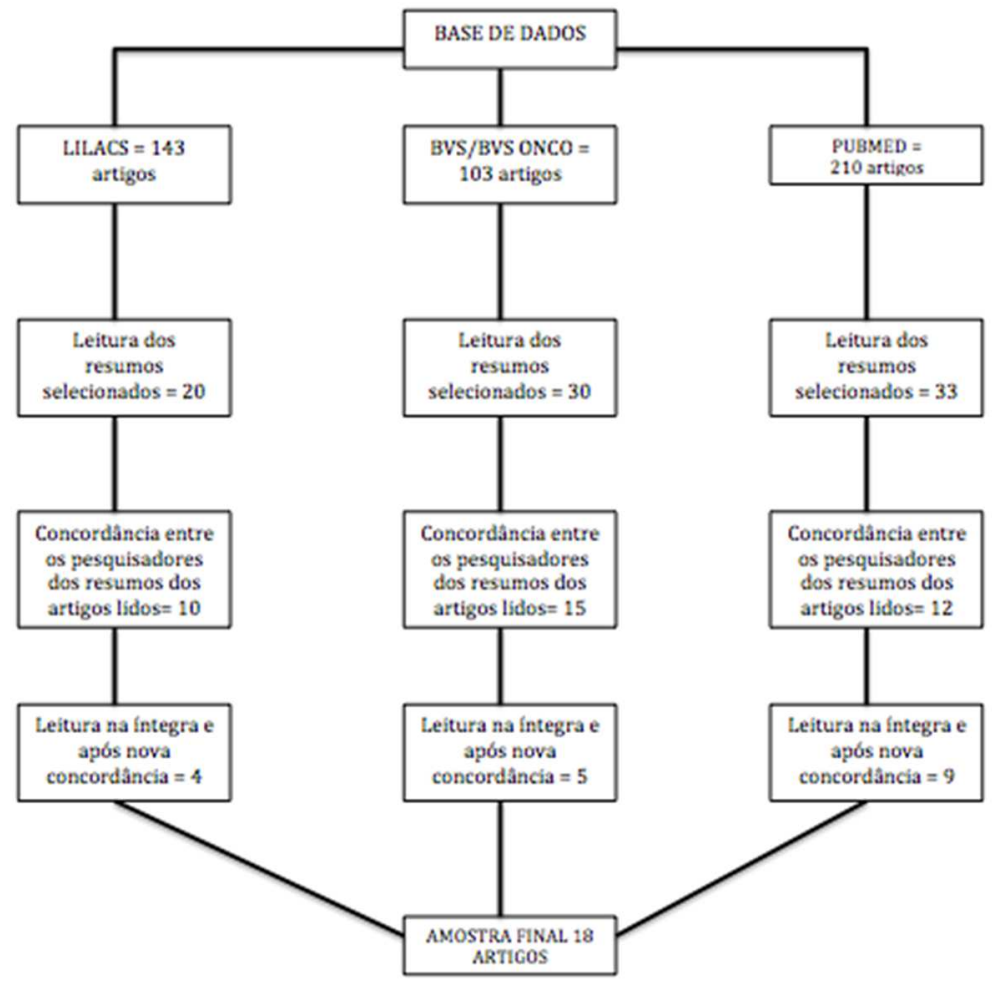

Fonte: dados da pesquisa. 


\section{Resultados e Discussão}

Tabela 1. Tabela expositiva dos artigos pesquisados neste estudo. Rio de Janeiro, RJ, Brasil, 2017.

\begin{tabular}{|c|c|c|}
\hline AUTOR/ANO & TÍTULO & OBJETIVOS \\
\hline $\begin{array}{l}\text { KARASOULI, E et al/ } \\
2016\end{array}$ & $\begin{array}{l}\text { Qualitative critical incidente } \\
\text { study of patient's experiences } \\
\text { leading to emergency hospital } \\
\text { admission with advanced } \\
\text { respiratory illness. }\end{array}$ & $\begin{array}{l}\text { Explorar as experiências de } \\
\text { pacientes com câncer respiratório } \\
\text { avançado, seus cuidadores e } \\
\text { profissionais de saúde, dentro da } \\
\text { emergência do hospital. }\end{array}$ \\
\hline $\begin{array}{l}\text { WANG, X. e CAO, H/ } \\
2015\end{array}$ & $\begin{array}{l}\text { A meta-analysis of } \\
\text { comprehensive care on quality of } \\
\text { life in patients with lung câncer. }\end{array}$ & $\begin{array}{l}\text { Avaliar a eficácia de cuidados } \\
\text { abrangentes sobre a qualidade de } \\
\text { vida em pacientes com câncer de } \\
\text { pulmão. }\end{array}$ \\
\hline $\begin{array}{c}\text { KAO YH, CHIANG, } \\
\text { JK/ } 2015\end{array}$ & $\begin{array}{l}\text { Effects of hospice care on quality } \\
\text { indicators of end-of-life care } \\
\text { among patients with liver } \\
\text { câncer: a natiobal longitudinal } \\
\text { population-basedstudyin Taiwan } \\
\text { 2000-2011. }\end{array}$ & $\begin{array}{l}\text { Avaliar o efeito do cuidado para } \\
\text { pacientes com câncer de fígado } \\
\text { avançado dentre admissões } \\
\text { hospitalares, terapias } \\
\text { emergências. }\end{array}$ \\
\hline $\begin{array}{l}\text { HENSON, LA et al/ } \\
2015\end{array}$ & $\begin{array}{l}\text { Factors associated with } \\
\text { agressive end of life câncer care. }\end{array}$ & $\begin{array}{l}\text { Investigar os fatores sócio- } \\
\text { demográficos, clínicos e } \\
\text { comunitários de cuidados de saúde } \\
\text { associados ao cuidado agressivo do } \\
\text { câncer de fim de vida. }\end{array}$ \\
\hline RAST, AC. et al/2015 & $\begin{array}{l}\text { Procalcitonin improves the } \\
\text { Glasgow prognostic score for } \\
\text { outcomeprediction in emergency } \\
\text { patients with cancer: a cohort } \\
\text { study. }\end{array}$ & $\begin{array}{l}\text { Validar o índice prognóstico de } \\
\text { Glasgow para prevenção da } \\
\text { mortalidade em pacientes com } \\
\text { alguma urgência médica } \\
\text { relacionada ao câncer. }\end{array}$ \\
\hline $\begin{array}{l}\text { BAKITAS, MA. et al/ } \\
2015\end{array}$ & $\begin{array}{l}\text { Early versus delayed initiation of } \\
\text { concurrent palliative oncology } \\
\text { care: patient outcomes in the }\end{array}$ & $\begin{array}{l}\text { Acompanhar mensalmente os } \\
\text { cuidados de pacientes com câncer } \\
\text { avançado em serviços como }\end{array}$ \\
\hline
\end{tabular}




\begin{tabular}{|c|c|c|}
\hline & $\begin{array}{l}\text { ENABLE III Randomized } \\
\text { controlled trial. }\end{array}$ & $\begin{array}{l}\text { emergência, terapia intensiva e } \\
\text { unidades de internação. }\end{array}$ \\
\hline $\begin{array}{l}\text { BRYANT, AL. et al/ } \\
2015\end{array}$ & $\begin{array}{l}\text { Use of ED and hospital services } \\
\text { for patients with acute leucemia } \\
\text { after induction therapy: one-year } \\
\text { follow-up. }\end{array}$ & $\begin{array}{l}\text { Examinar os principais motivos de } \\
\text { admissão no departamento de } \\
\text { emergência relacionado ao } \\
\text { atendimento de pacientes com } \\
\text { leucemia aguda após terapia de } \\
\text { indução até um ano após a alta. }\end{array}$ \\
\hline RUEGG, TA. 2013 & $\begin{array}{l}\text { A nurse practitioner-led urgente } \\
\text { care center: meeting the need } \\
\text { sof the patient with cancer. }\end{array}$ & $\begin{array}{l}\text { Descrever um centro de } \\
\text { atendimento de urgência para } \\
\text { pacientes com câncer, liderado por } \\
\text { enfermeiras. }\end{array}$ \\
\hline LEONARD, K. 2012 & $\begin{array}{l}\text { A European survey relating to } \\
\text { câncer therapy and neutropenic } \\
\text { infections: nurse and patients } \\
\text { view points. }\end{array}$ & $\begin{array}{l}\text { Avaliar as perspectivas de } \\
\text { enfermeiros sobre a prevenção de } \\
\text { infecção em pacientes que } \\
\text { recebem quimioterapia, evitando } \\
\text { emergências oncológicos. }\end{array}$ \\
\hline $\begin{array}{l}\text { ALVES, NLA. et al/ } \\
2012\end{array}$ & $\begin{array}{l}\text { Assistência de enfermagem nas } \\
\text { emergências oncológicas } \\
\text { decorrentes das toxicidades do } \\
\text { tratamento oncológico }\end{array}$ & $\begin{array}{l}\text { Analisar a assistência de } \\
\text { enfermagem nas emergências } \\
\text { oncológicas decorrentes das } \\
\text { toxicidades do tratamento } \\
\text { quimioterápico. }\end{array}$ \\
\hline $\begin{array}{l}\text { MANZI, NM. et al./ } \\
2012\end{array}$ & $\begin{array}{l}\text { Intervenções de enfermagem } \\
\text { relacionadas ao tratamento das } \\
\text { emergências } \\
\text { sindrômicas }\end{array}$ & $\begin{array}{l}\text { Informar sobre as emergências } \\
\text { oncológicas e as principais } \\
\text { intervenções de enfermagem } \\
\text { relacionadas presentes na } \\
\text { literatura. }\end{array}$ \\
\hline $\begin{array}{l}\text { PINHEIRO, APB. et } \\
\text { al/2011 }\end{array}$ & $\begin{array}{l}\text { Uma reflexão sobre o cuidado de } \\
\text { enfermagem na emergência } \\
\text { oncológica }\end{array}$ & $\begin{array}{l}\text { Contribuir com a reflexão acerca do } \\
\text { cuidado de enfermagem nos } \\
\text { serviços de emergência } \\
\text { hospitalares ao cliente acometido } \\
\text { com câncer. }\end{array}$ \\
\hline
\end{tabular}




\begin{tabular}{|c|c|c|}
\hline $\begin{array}{l}\text { PEIXOTO, IC. et al./ } \\
2011\end{array}$ & $\begin{array}{l}\text { Análise do perfil dos pacientes } \\
\text { oncológicos sem possibilidades } \\
\text { terapêuticas de cura atuais: } \\
\text { verificação da demanda por } \\
\text { cuidados paliativos em hospital } \\
\text { universitário }\end{array}$ & $\begin{array}{l}\text { Categorizar os pacientes em } \\
\text { cuidados paliativos oncológicos, } \\
\text { analisar esta demanda de acordo } \\
\text { com o tipo de câncer e grau de } \\
\text { estadiamento; e identificar as } \\
\text { principais síndromes } \\
\text { paraneoplásicas existentes nestes } \\
\text { pacientes. }\end{array}$ \\
\hline $\begin{array}{l}\text { GOMES, A.R. et al./ } \\
2010\end{array}$ & $\begin{array}{l}\text { Demanda potencial de um } \\
\text { serviço de emergência pediátrica } \\
\text { oncológica: a atuação do } \\
\text { enfermeiro }\end{array}$ & $\begin{array}{l}\text { Identificar a demanda potencial de } \\
\text { pacientes atendidos no Serviço de } \\
\text { Emergência Pediátrica Oncológica } \\
\text { do Instituto Nacional do Câncer. }\end{array}$ \\
\hline CALIL, AM./ 2010 & $\begin{array}{l}\text { Avaliação de pacientes } \\
\text { oncológicos na emergência: uma } \\
\text { revisão de literatura }\end{array}$ & $\begin{array}{l}\text { Identificar condições clínicas e/ou } \\
\text { cirúrgicas prevalentes nos } \\
\text { pacientes que procuram o serviço } \\
\text { de emergência oncológica. }\end{array}$ \\
\hline SMITH, LH./ 2009 & $\begin{array}{l}\text { Emergency Response in } \\
\text { Outpatient Oncology Care: } \\
\text { Improving Patient Safety }\end{array}$ & $\begin{array}{l}\text { Analisar a participação do } \\
\text { enfermeiro em um grupo } \\
\text { especializado e multidisciplinar, } \\
\text { para o cuidado do paciente } \\
\text { oncológico na emergência. }\end{array}$ \\
\hline $\begin{array}{l}\text { PIGNATARI, SC. et } \\
\text { al/ } 2008\end{array}$ & $\begin{array}{l}\text { Emergências oncológicas: } \\
\text { Assistência de enfermagem } \\
\text { proposta na literatura }\end{array}$ & $\begin{array}{l}\text { Identificar o conhecimento sobre a } \\
\text { assistência de enfermagem nas } \\
\text { emergências oncológicas para } \\
\text { rápidas intervenções. }\end{array}$ \\
\hline $\begin{array}{l}\text { DE CAMARGOS, MG. } \\
\text { et al./ } 2007\end{array}$ & $\begin{array}{l}\text { Atuação do enfermeiro frente às } \\
\text { principais emergências } \\
\text { oncológicas }\end{array}$ & $\begin{array}{l}\text { Descrever as principais } \\
\text { emergências oncológicas e a } \\
\text { atuação do enfermeiro frente às } \\
\text { mesmas. }\end{array}$ \\
\hline
\end{tabular}

Fonte: dados da pesquisa.

No levantamento dos artigos científicos, observa-se pouca quantidade de material de pesquisa voltado para a assistência de enfermagem. Verificou-se que, em alguns casos, os cuidados de enfermagem ao paciente oncológico emergencial estavam inseridos em artigos voltados para o assunto de cuidados paliativos. 
As principais emergências e intervenções abordadas nos estudos são: síndrome da veia cava superior, com a avaliação da frequência respiratória e elevação da cabeceira do paciente; síndrome da lise tumoral, enfocando a avaliação cardíaca, neurológica e gastrointestinal; compressão medular, atentando para a semiologia da dor e mobilidade do paciente; hipercalcemia e hiponatremia, salientando a atenção quanto à hidratação e à reposição de eletrólitos e monitoramento do acesso venoso; e neutropenia febril, com ênfase no exame físico para achado de focos infecciosos, administração correta de antibióticos e cuidados com dispositivos de infusão.

\section{Categoria 1- A enfermagem na emergência} oncológica: principais sinais e sintomas

As emergências oncológicas classificam-se como: metabólicas, neurológicas, cardiovasculares, hematológicas, respiratórias e urológicas, ou estarem relacionadas aos efeitos colaterais do tratamento(4). Uma subclassificação dessas emergências poderia ser realizada da seguinte forma: emergências estruturais ou obstrutivas (causadas pela compressão da massa tumoral às estruturas corpóreas), emergências metabólicas e emergências secundárias ao tratamento. Algumas têm início insidioso, levando meses para desenvolverem-se, enquanto outras manifestam-se em horas, causando resultados devastadores como paralisia e morte ${ }^{(5)}$.

As emergências oncológicas mais comuns são relacionadas à Síndrome de compressão medular, representando uma emergência oncológica que geralmente ocorre com o crescimento da tumoração na região epidural(6). Na Síndrome da veia cava superior, a obstrução da veia cava superior pode ser causada por trombose, compressão extrínseca ou invasão direta da veia e, frequentemente, por uma associação destes fatores $^{(7)}$. O correto posicionamento do paciente, com a cabeça e os membros inferiores elevados, otimiza o conforto respiratório. O controle hídrico rigoroso, a manutenção do repouso absoluto, o controle hidroeletrolítico, e o manejo contínuo da dor são alguns dos cuidados indispensáveis nestas emergências.

Os cânceres mais comuns associados a hipercalcemia, são: mama, pulmão e mieloma múltiplo. Emergências metabólicas como a hipercalcemia são relativamente comuns em pacientes com câncer, ocorrendo em aproximadamente de 20 a $30 \%$ dos casos no desenvolvimento da doença. Os pacientes com câncer também sofrem de hiponatremia e a fisiopatologia nem sempre é compreendida. Trata-se de uma alteração eletrolítica frequente na prática clínica, a qual 
reflete uma alteração do balanço de água, com consequente diminuição da osmolaridade plasmática(8). O controle da hidratação venosa e a avaliação do padrão respiratório visam diminuir o desconforto do paciente e auxiliar no tratamento deste distúrbio.

Outro sintoma frequente é a falta de ar, sendo um dos motivos que levam os pacientes a procurar o serviço de emergência oncológica(28). Pode ser observada também em pacientes que possuem comorbidades, como a Doença Pulmonar Obstrutiva Crônica (DPOC). A falta de ar também ocorre devido a obstruções de fluxo sanguíneo, causadas pela compressão dos tumores. Os pacientes com câncer de pulmão procuram as emergências com queixas de dor, causadas muitas vezes pelo comprometimento respiratório(28). A avaliação da escala de dor e administração de analgesia prescrita favorecem o conforto do paciente e o controle da evolução do quadro clínico.

A Síndrome de Lise Tumoral (SLT) caracteriza-se pela destruição maciça de células malignas. Tal síndrome está muitas vezes associada ao Linfoma Não-Hodgkin (LNH), particularmente, o linfoma de Burkitt e leucemias (Leucemia Mieloide Aguda e Leucemia Linfocítica Aguda), após o início da quimioterapia ${ }^{(8)}$. Orientar quanto a hidratação, 24 a 48 horas antes da terapia citotóxica auxilia na prevenção da emergência e na possibilidade de uma insuficiência renal.

\section{Emergências secundárias ao tratamento oncológico}

A neutropenia febril é uma das principais complicações associadas ao tratamento quimioterápico. O risco de desenvolver tal emergência depende da profundidade e duração do NADIR (tempo transcorrido entre a aplicação da droga e o aparecimento do menor valor de contagem hematológica dos neutrófilos, ocorrendo em média de 5 a 10 dias após a última dose de quimioterapia), e da intensidade e duração do regime quimioterápico, assim como das comorbilidades e complicações, como as mucosites $^{(4-9-32)}$.

Neste sentido, os enfermeiros devem conhecer os conceitos sobre a doença, seus aspectos biológicos e fatores que influenciam no contexto da emergência oncológica, além de saber reconhecer os principais sinais e sintomas desse tipo de emergência. Assim, poderão atuar em diversos níveis de atenção à saúde, segundo o grau de complexidade, desenvolvendo uma assistência de enfermagem de qualidade aos pacientes oncológicos $^{(10)}$. Destaca-se no estudo ${ }^{(11)}$, uma série de condições clínicas que motivam a busca do paciente com algum tipo de afecção 
oncológica ao hospital, como por exemplo: quadros infecciosos, desidratação, obstrução intestinal, anemias, distúrbios metabólicos, entre outros. Logo, apontam-se importantes lacunas de conhecimento na literatura nacional.

\section{A detecção precoce destas} condições clínicas, seus sinais e sintomas apresentados e as orientações de enfermagem como melhora da ingesta hídrica, controle no uso de analgesia, informações sobre as reações adversas da radioterapia e quimioterapia, dada aos pacientes e familiares, fazem com que uma resposta satisfatória ao tratamento oncológico nas emergências prossiga de forma ideal para uma melhora progressiva da saúde do paciente com agravos emergenciais oncológicos ${ }^{(12)}$.

Uma revisão sobre as emergências oncológicas mais comuns ainda é realizada com maior ênfase na prevenção, sinais de alarme, diagnóstico e tratamento. Tais emergências representam uma variedade de situações que podem ocorrer a qualquer altura durante o curso da doença maligna. Com isso, é importante que todos os profissionais que cuidam de pessoas que vivem com câncer, tenham um conhecimento sólido das principais emergências oncológicas e estejam atentos aos sinais de alarme de cada situação específica(4).
Aborda-se também, neste estudo(13), a SLT, afirmando-se que a correção dos sintomas facilita o alívio rápido das alterações metabólicas. Ressalta-se que os fatores de risco para esta condição devem ser prontamente reconhecidos, para que a profilaxia e o tratamento adequados sejam instituídos ${ }^{(14)}$.

Esta questão é particularmente importante para pacientes pediátricos, já que cerca de $70 \%$ pode ser curado com regime quimioterápico adequado. Além disso, os casos de lise tumoral espontânea, embora menos comuns, são particularmente críticos, visto que muitas vezes apresentam-se em crianças que ainda não estão sob os cuidados de especialistas, destacando a necessidade de que enfermeiros pediatras, clínicos e intensivistas também tenham o conhecimento e a segurança essenciais para a boa condução dos $\operatorname{casos}^{(14)}$.

Os profissionais dos setores de emergência lidam com os diferentes sintomas de pacientes com câncer. No entanto, as emergências oncológicas com grande demanda de pacientes, podem não ser os melhores ambientes para pacientes com câncer, pois a neutropenia aumenta o risco de desenvolver outras infecções bacterianas, virais e fúngicas ${ }^{(34)}$.

\section{Categoria 2- Cuidado de enfermagem nas} emergências oncológicas: ver, ouvir e intervir 
Em relação aos artigos pesquisados, verificou-se que dos 18 artigos, apenas 03 tratavam do cuidado integral, aquele que abrange também o binômio paciente-família. A família faz parte da sustentação da vida do paciente oncológico. A parte biopsicossocial deve ser compreendida de forma integral, e os profissionais necessitam estar preparados para atender as questões emocionais, psicológicas e sociais desta clientela específica $^{(15)}$.

A intervenção rápida da equipe faz-se necessária para evitar a morte ou uma lesão permanente e grave. Ressalta-se que é por meio da comunicação que identificam-se os problemas e as necessidades do cliente. Por conseguinte, um dos desafios no atendimento de emergência é saber ouvir adequadamente para poder intervir, de acordo com as expectativas da pessoa e da família. Além do conhecimento técnico das emergências, a equipe precisa ter domínio sobre a questão emocional e social, tanto para a clientela quanto para os familiares, a fim de realizar um cuidado de qualidade ${ }^{(16)}$.

São importantes também, as intervenções de enfermagem relacionadas às emergências oncológicas sindrômicas, como: os cuidados referentes à dor e a administração correta de analgésicos e de anti-inflamatórios. Ressalta-se ainda a importância da mudança de decúbito e dos benefícios que tal procedimento proporciona, melhorando a compressão de alguns órgãos e aliviando as queixas álgicas. Tal conhecimento técnico e científico, juntamente com o apoio emocional e as orientações para o domicílio, é importante na efetividade das ações para com a clientela assistida, melhorando o bem-estar físico do paciente oncológico e contribuindo para a qualidade de vida dos indivíduos ${ }^{(5-35)}$.

De modo geral, existe um pouco conhecimento sobre $\mathrm{o}$ atendimento desta clientela específica, e a demanda de atendimento nas unidades de alta complexidade tem crescido. A implantação de um plano de cuidados também torna-se importante na resolução dos problemas apresentados, os quais foram coletados por meio da entrevista e do histórico de enfermagem $^{(17)}$.

Outros artigos abordavam o cuidado de enfermagem frente às emergências oncológicas, voltado especificamente para a parte técnica, não abrangendo aspectos subjetivos do cuidado, tais como: as relações familiares e estratégias no acompanhamento do paciente oncológico. A interpretação de marcadores sanguíneos fornece informações úteis que melhoram o gerenciamento e a tomada de decisão durante um atendimento de enfermagem na emergência, principalmente se a emergência oncológica 
em questão for uma neutripenia febril, mas os aspectos subjetivos são igualmente importante $^{(32)}$.

Um dos estudos(18), aborda a ida do paciente oncológico ao setor de emergência, devido as intensas alterações decorrentes das toxicidades do tratamento quimioterápico, sendo de responsabilidade da equipe de enfermagem prestar a assistência inicial. 0 autor afirma que a assistência de enfermagem ao paciente oncológico é um desafio e requer um grande conhecimento sobre as diversas técnicas e procedimentos. Comentando ainda sobre mudanças no cotidiano das famílias, que sempre têm muitas dúvidas sobre qual é o momento exato para procurar o serviço de emergência.

Os cuidados de enfermagem são executados interdependentemente de acordo com a prescrição e a orientação do médico. Considerando-se que os enfermeiros estão habilitados para avaliar e identificar os problemas de assistência à saúde do paciente em situações de crise, sobretudo em emergências oncológicas. Tais intervenções estão voltadas para a observação do paciente, tanto de suas condições físicas quanto de parâmetros fisiológicos; favorecimento do conforto físico; orientação do paciente e seus familiares sobre os mais diversos aspectos; manejo da dor, de lesão tecidual, alterações metabólicas, hemodinâmicas e hematológicas ${ }^{(19)}$.

$$
\text { Um dos estudos }{ }^{(20)} \text { enfatizou o }
$$

cuidado na síndrome de compressão medular, com abordagem nos cuidados técnicos nesta situação de emergência. Citase a importância da interpretação dos principais sinais apresentados por estes pacientes, tais como a dor, recomendando a mudança de decúbito para o alívio da compressão local e a transmissão de orientações aos familiares.

Outro artigo $^{(21)}$ esclareceu a importância da atuação do enfermeiro frente à demanda em um pronto atendimento oncológico pediátrico. Foi reforçado o conhecimento técnico das patologias mais comuns para um bom desempenho no atendimento dessa clientela.

Reforçou-se ainda, que o principal elemento no atendimento das urgências metabólicas está na capacidade de interpretação dos exames laboratoriais e na rápida correção dos distúrbios hidroeletrolíticos ${ }^{(8)}$. No caso do quadro clínico de neutropenia febril, ressaltou-se a importância do uso de antibióticos de largo espectro, favorecendo uma melhor resposta aos tratamentos com quimioterápicos mais $\operatorname{agressivos}^{(23)}$.

Um dos $\operatorname{artigos}^{(18)}$ encontrados, 
discorre sobre o cuidado de enfermagem nas emergências oncológicas relativo ao tratamento quimioterápico. $\mathrm{O}$ autor acredita que é de grande relevância procurar novos métodos e estratégias que auxiliem na assistência de enfermagem a estes pacientes, tentando minimizar sua dor e seu sofrimento. Como dito anteriormente, não basta conhecer o cuidado literalmente, mas saber aplicá-lo qualitativamente.

Desta forma, todos os profissionais de saúde devem reavaliar suas condutas perante os pacientes oncológicos, tendo em vista que um dos objetivos principais do tratamento é a manutenção da dignidade humana. Sugere-se a criação de um instrumento de articulação entre as diversas categorias de saúde, semelhante à coleta de dados, favorecendo a integração de informações, fazendo com que cheguem mais completas às equipes de saúde, e que os profissionais possam conhecer os cuidados em sua totalidade e saber aplicá-los em prol da melhoria de saúde do indivíduo ${ }^{(17)}$.

O quantitativo de estudos sobre as intervenções de enfermagem na área oncológica ainda é pequeno. Alguns profissionais sabem da importância dos cuidados, mas não conseguem aplicá-los durante os atendimentos, fazendo com que a qualidade das ações caia e o atendimento torne-se mecanizado, executando-se tarefas de modo repetitivo ${ }^{(19)}$.

Independente de qual seja a situação emergencial e esperando que o profissional de enfermagem tenha conhecimento a respeito das condutas a serem providenciadas, torna-se importante que também haja bom senso, dedicação, respeito, agilidade, assepsia, cuidados com autoproteção e técnica, para garantir um ambiente seguro e eficaz nas urgências ou emergências. Entretanto, algumas instituições de graduação em saúde não abordam o tema de forma aprofundada, deixando a desejar na preparação do profissional para atender uma das doenças, que consiste na maior incidência na população mundial(10).

É ressaltada a importância do conhecimento aprofundado das principais emergências oncológicas pelas diversas equipes de saúde, as quais devem estar atentas aos sinais de alarme das inúmeras patologias $^{(4)}$. A opinião de equipes multidisciplinares no atendimento das emergências oncológicas é um fator crucial para o bom atendimento e um melhor prognóstico deste público específico ${ }^{(24)}$.

Durante 0 atendimento de emergência, em um hospital geral, muitas vezes, torna-se difícil avaliar o paciente oncológico e tratar suas complicações. Diante disso, surge a necessidade de promover estudos e revisões técnicas sistemáticas, para 
que aspectos emergenciais sejam abordados de modo que um bom prognóstico e uma melhoria da qualidade de vida sejam alcançados como resultados finais ${ }^{(35)}$.

Salienta-se que quanto mais rápido e melhor forem abordados estes assuntos com os pacientes, mais precocemente retornarão às suas rotinas de vida. Fala-se ainda que $o$ conhecimento das patologias oncológicas auxilia nos cuidados e no tratamento ${ }^{(21)}$.

\section{Categoria 3 - Os Cuidados paliativos de enfermagem nas emergências oncológicas}

Para pacientes com câncer avançado, as visitas aos setores de emergência são comuns. Esses pacientes apresentam necessidades de cuidados paliativos desencadeados do agravamento do câncer. Queixas como dor, falta de ar ou vômitos, que não podem ser controlados e acabam fragilizando o indivíduo.

O cuidado paliativo utiliza uma abordagem interdisciplinar, colaborativa e baseada em equipe para diminuir dor e sofrimento para pacientes com doença avançada. Nestes casos, o objetivo é alcançar a melhor qualidade possível de vida, incluindo aspectos físicos, psicológicos, sociais e espirituais, para pacientes e famílias, através de conhecimento e habilidades específicas. Este cuidado não limita-se aos cuidados de fim de vida e, é oferecido simultaneamente com terapias de prolongamento da vida, para pessoas que vivem com doenças crônicas graves. Demonstrou-se que melhora significativamente a qualidade de vida dos pacientes e membros da família, ao mesmo tempo que reduz os custos de serviços de saúde(37).

A qualidade de vida dos indivíduos que procuram os serviços de emergência melhora não somente com benefícios ao sinais e sintomas no corpo humano oferecidos pelos profissionais, mas também na questão do humor. A sobrevivência do indivíduo, na maioria das vezes, se dá pelo seu bem estar social. O cuidado nas emergências oncológicas não é somente uma questão técnica, mas também uma forma de olhar o indivíduo em sua totalidade ${ }^{(33)}$.

Melhorar a qualidade dos pacientes com câncer é muito importante especialmente para pacientes não curáveis. Ao criar um bom ambiente para o paciente durante a recepção na emergência, causa uma melhora das funções físicas e da função emocional. Além disso, este atendimento e esta recepção variam muito de um indivíduo a outro, devido características demográficas culturais e sociais. $\mathrm{O}$ cuidado de enfermagem na visão da totalidade, causa a diferença na vida de pacientes e familiares ${ }^{(30)}$. 


\section{Conclusão}

O enfermeiro deve estar habilitado para avaliar e identificar os problemas ocasionados pelas emergências oncológicas. Intervenções de enfermagem como: observação do paciente, tanto de suas condições físicas como de parâmetros fisiológicos; favorecer o conforto físico; orientar o paciente e seus familiares sobre os mais diversos aspectos; manejo da dor, de lesão tecidual, alterações metabólicas, hemodinâmicas e hematológicas, são realizadas com o intuito de salvaguardar a integridade do indivíduo.

No entanto, o conhecimento identificado neste estudo sobre a assistência de enfermagem nas emergências oncológicas nas publicações selecionadas mostrou-se reduzido. Todavia, entre os artigos pesquisados, a maioria apresentou intervenções de enfermagem específicas para cada situação. O binômio paciente-família deve ser bem acompanhado durante uma emergência, para que o paciente seja visto tanto na esfera biológica quanto na psicológica, cultural e social.

As intervenções de enfermagem são executadas interdependentemente de acordo com a prescrição e a orientação do médico.
Considerando-se que a equipe está habilitada para recepcionar esse tipo de paciente, avaliando e identificando os problemas apresentados em emergências oncológicas, faz-se necessário que haja um maior incentivo para essa área de pesquisa, para que os profissionais fiquem atualizados e embasados em princípios científicos, alcançando assim um bom desempenho. $\mathrm{O}$ estímulo ao desenvolvimento de pesquisas e o treinamento da equipe melhoram o aproveitamento das práticas do enfermeiro.

Pretendeu-se com este estudo, contribuir para o aumento do conhecimento referente ao cuidado de enfermagem com o paciente na emergência oncológica, possibilitando a realização de novas pesquisas sobre a temática, demonstrando assim, que a qualidade assistência prestada é fundamental e faz diferença nas práticas de saúde.

A contribuição social fica por conta do atendimento emergencial nestes casos, realizado com eficácia, trazendo melhorias significativas para $o$ paciente e seus familiares, facilitando o retorno do mesmo para seu cotidiano. A contribuição para o ensino de enfermagem se dará pelo envolvimento de estudantes de graduação e pós-graduação neste cenário de prática, enriquecendo 0 saber científico do enfermeiro e acarretando mudanças em sua formação profissional. 


\section{Referências}

1. Brasil. Ministério da Saúde. Secretaria de Atenção à Saúde. Departamento de Regulação, Avaliação e Controle. Coordenação Geral de Sistemas de Informação. Manual de Bases Técnicas da Oncologia- SIA/SUS- Sistemas de informações ambulatoriais. 21 edição. 135p. Set 2015.

2. Instituto Nacional de Câncer. A situação do Câncer no Brasil. Rio de Janeiro, 2008.

3. Mendes KDS, Silveira RCCP, Galvão CM. Revisão integrativa: método de pesquisa para a incorporação de evidências na saúde e na enfermagem. Texto Contexto Enferm [Internet]. 2008 [acesso em 25 nov 2011]; 17(4). Disponível em: http://dx.doi.org/10.1590/S010407072008000400018.

4. Fortes OC. Emergências Oncológicas. Mestrado Integrado em Medicina. Instituto de Ciências Biomédicas Abel Salazar. Universidade do Porto. Porto. 2011.

5. Manzi NM et al. Intervenções de enfermagem relacionadas ao tratamento das emergências oncológicas sindrômicas. Journal of Nursing Online. 2007; 6(9):2307-2311.

6. Brunner LS, Suddarth DS. Tratado de Enfermagem Médico-Cirúrgica. 12a ed. Rio de Janeiro: Editora Guanabara Koogan, 2012.

7. Hoff PMG et al. Tratado de Oncologia. 1a ed. São Paulo: Atheneu, 2013.

8. Maradei S, Arcuri LJ. Urgências metabólicas no paciente oncológico. Rio de Janeiro: Onco\&., 2011.

9. Bonassa EMA, Gato MIR. Terapêutica Oncológica para enfermeiros e farmacêuticos. $4^{\text {a }}$ ed. São Paulo: Atheneu, 2012.
10. De Camargos MG et al. Atuação do enfermeiro frente as principais emergências oncológicas. In: XV Encontro Latino Americano de Iniciação Científica e XI Encontro Latino Americano de Pós Graduação- Universidade do Vale do Paraíba. 2007.

11. Calil AM. Avaliação de pacientes oncológicos na emergência: uma revisão de literatura. Nursing. 2010; 13(146):360-364.

12. Smith LH, Shiavone R. Emergency Response in Outpatient Oncology Care: Improving Patient Safety. Clinical Journal of Oncology Nursing. 2009; 13(4).

13. Tallo FS et al. Síndrome da lise tumoral: uma revisão para o clínico. Revista Brasileira de Clínica Médica. 2013; 11(2):150-154.

14. Ferraz ST. Síndrome de lise tumoral em Pediatria. Grupo Editorial Moreira Junior/Pediatria moderna. 2013; 49(1):19-21.

15. Gargiulo CA, Melo MCSC, Salimena AMO, Bara VMF, Souza IEO. Vivenciando o cotidiano do Cuidado na percepção de Enfermeiras Oncológicas. Texto Contexto Enferm. 2007; 16(4):696-702.

16. Pinheiro APB et al. Uma reflexão sobre o cuidado de enfermagem na emergência oncológica. Revista de Pesquisa: cuidado é fundamental. 2011; 3(1):1747-1752.

17. Peixoto IC et al. Análise do perfil dos pacientes oncológicos sem possibilidades terapêuticas de cura atuais: verificação da demanda por cuidados paliativos em hospital universitário. Rev HUPE. 2011; 10(Supl.1):5363.

18. Alves NLA et al. Assistência de enfermagem nas emergências oncológicas decorrentes das toxicidades do tratamento 
oncológico. In: Congresso Brasileiro dos Conselhos de Enfermagem. Fortaleza. 2012.

19. Pignatari SC, Silveira RCCP, De Carvalho EC. Emergências oncológicas: Assistência de enfermagem proposta na literatura. Online Brazilian Journal of Nursing. 2008; 7(3).

20. Feital RR. Emergências Oncológicas: atuação do técnico de enfermagem na Síndrome de Compressão Medular. In: Instituto Nacional do Câncer. Rio de Janeiro. 2010.

21. Gomes AR et al. Demanda potencial de um serviço de emergência pediátrica oncológica: a atuação do enfermeiro. Instituto Nacional do Câncer. Rio de Janeiro. 2010.

22. Guedes MVC, Henriques ACPT, Lima MMN. Acolhimento em um serviço de emergência: uma percepção dos usuários. Revista Brasileira de Enfermagem. 2013; 66(1).

23. Torres LG, Tabak D. Neutropenia Febril e Câncer-parte 1. Rio de Janeiro: Onco \&., 2011.

24. Cereceda LG. Emergências Oncológicas. Revista Médica Clínica Condes. 2011; 22:665676.

25. Instituto Nacional De Câncer. Ações de Enfermagem para o controle do câncer: uma proposta de integração ensino-serviço. 3a ed. Rio de Janeiro: INCA, 2008.

26. Leopardi MT. Metodologia da Pesquisa na Saúde. Santa Maria: Palloti, 2001.

27. Junior JAB, Matsuda LM. Construção e validação de instrumento para avaliação do acolhimento com classificação de risco. Revista Brasileira de Enfermagem. 2012; 65(5).
28. Karasouli E, Munday D, Bailey C, Staniszewska S, Hewison Alistair and Griffiths F. Qualitative critical incident study of patients'experiences leading to emergency hospital admission with advanced respiratory illness. BMJ Open. 2016.

29. Henson LA, Gomes B, Koffman J, Daveson BA, Higginson IJ, Gao W. Factors associated with aggressive end of life cancer care. Support Care Cancer. 2016; 24:1079-1089.

30. Wang X, Cao H. A meta-analysis of comprehensive care on quality of life in patients with lung cancer. Cancer Journal. 2017.

31. Kao YH, Chiang JK. Effect of hospice care on quality indicators ofend-of-life care among patients with liver cancer: a national longitudinal population-based study in Taiwan 2000-2011. BMC PalliativeCare. 2015; 14:39.

32. Rast AC, Kutz A, Felder S, Faessler L, Steiner D, Laukemann S, Haubitz $S$ et al. Procalcitonin Improves the Glasgow Prognostic Score for Outcome Prediction in Emergency Patients with Cancer: A Cohort Study. Hindawi Publishing Corporation; Disease Markers. 2015.

33. Bakitas MA, Tosteson TD, Li Z, Lyons KD, Hull JG, Dionne-Odom JN et al. Early Versus Delayed Initiation of Concurrent Palliative Oncology Care: Patient Outcomes in the ENABLE III Randomized Controlled Trial. J ClinOncol. 2015; 33:1438-1445.

34. Bryant AL, Deal AM, Walton A, Wood W, Muss $H$, Mayer DK. Use of ED and Hospital Services for Patients with Acute Leukemia after Induction Therapy: One-year Follow-Up. Leuk Res. 2015; 39(4):406-410.

35. Ruegg TA. A Nurse Practitioner-Led Urgent Care Center: Meeting the Needs of the 
Patient with Cancer. Clinical Journal of Oncology Nursing. 2013; 17(4).

36. Leonard KA. European survey relating to cancer therapy and neutropenic infections: Nurse and patient viewpoints. European Journal of Oncology Nursing. 2012; 16:380386.

37. Kandarian B, Morison RS, Richardson LD, Ortiz J, Grundzen CR. Emergency departmentinitiated palliative care for advanced câncer patients: protocol for a pilot randomized controlled trial. Trials Journal. 2014; 15(251).

38. Anjos ACY, Zago MMF. A experiência da terapêutica quimioterápica oncológica na visão do paciente. Rev. Latino-Am. Enferm. 2006; 14(1):33-40. 